\title{
Teoria da Mente e Desenvolvimento Social na Infância
}

\author{
Theory of Mind and Social Development in Childhood
}

Renata de Lourdes Miguel da Silva

Marisa Cosenza Rodrigues ${ }^{\mathrm{I}}$

Flávia Fraga Silveira

Resumo

A capacidade de compreender os estados mentais, que permite predizer e explicar as açóes humanas por meio da linguagem desenvolve-se gradualmente na infância. A aquisição dessa capacidade tem um impacto direto sobre o desenvolvimento social das crianças, em especial nas relaçóes com seus pares. Nesta direção, destacam-se aspectos como as relaçóes de amizade, o comportamento pró-social, a empatia e a aceitação social. O presente artigo apresenta e discute estudos nacionais e internacionais que investigaram as possíveis relaçóes entre o desenvolvimento social e a compreensão dos estados mentais, tais como desejos, crenças e emoçóes.

Palavras-chave: Desenvolvimento sociocognitivo; aceitação social; interação social; teoria da mente.

\section{Abstract}

The ability to understand mental states that enables us to predict and explain human actions through language develops gradually during childhood. The acquisition of this ability has a direct impact on the social development of children, especially in their peer relations. In this sense, such aspects like friendship, pro-social behavior, empathy and social acceptance are discussed. This article presents and analyzes some national and international studies that have investigated the possible relationship between social development and the understanding of mental states such as desires, beliefs and emotions.

Keywords: Socio-cognitive development; social acceptance; social interaction; theory of mind.

Universidade Federal de Juiz de Fora

Nas três últimas décadas, a Psicologia iniciou estudos que investigam a habilidade infantil de compreender e predizer o comportamento próprio e alheio por meio da atribuição de estados mentais, tais como desejo, emoção e crença, área denominada teoria da mente.

Os estudos pioneiros (e.g. Baron-Cohen, Leslie \& Frith, 1985; Flavell, Flavell \& Green, 1983; Wimmer \& Perner, 1983) dedicaram especial atenção para a aquisiçáo e o desenvolvimento desta habilidade sociocognitiva. Nesta direção também podem ser encontradas pesquisas nacionais realizadas nos anos 1990 (e.g., Dias, 1993; Dias, Soares \& Sá, 1994). Como informam Flavell, Miller e Miller (1999), a gama de investigaçóes conduzidas em realidades culturais distintas indica que as crianças com desenvolvimento típico, por volta dos quatro anos obtêm sucesso em tarefas de teoria da mente.

A área de estudos em questão vem ganhando destaque no cenário da Psicologia Cognitiva e do
Desenvolvimento, especialmente em investigaçôes que contemplam, por exemplo, as possíveis interfaces entre teoria da mente e linguagem (Astington \& Jenkins, 1999; Harris, Rosnay \& Pons, 2005). Tais investigaçôes evidenciam que as habilidades linguísticas relacionam-se à compreensão dos estados mentais. Destacam-se também pesquisas contemplando as possíveis relaçóes entre teoria da mente e o desenvolvimento social, a partir das quais vem sendo evidenciada relaçáo entre o desempenho em tarefas de avaliação da teoria da mente e a manifestaçáo de comportamentos positivos com pares (Cassidy, Werner, Rourke, Zubernis \& Balaraman, 2003; Watson, Nixon, Wilson \& Capage, 1999).

Este artigo apresenta e discute estudos que investigaram o desenvolvimento da teoria da mente e suas possíveis relaçôes com aspectos relevantes do desenvolvimento social infantil, tais como a aceitação pelos pares, a pró-sociabilidade e a habilidade de fazer amizades. 


\section{A Importância da Teoria da Mente para o Desenvolvimento Social}

Em sua trajetória de desenvolvimento, a criança adquire progressivamente a habilidade de compreender crenças, desejos e emoçôes (Wellman \& Liu, 2004). Como argumentaram Lyra, Roazzi e Garvey (2008), essa aquisição é de fundamental importância para a sua inserção no mundo social, visto que a teoria da mente está estritamente relacionada ao estabelecimento, manutenção e êxito das relaçóes sociais.

Pavarini, Loureiro e Souza (2011) afirmam que crianças mais atentas aos desejos, sentimentos e pensamentos alheios tendem a ser melhor avaliadas socialmente do que aquelas que não reconhecem esses estados internos tão prontamente. As autoras mencionam ainda que crianças com maior aceitação social tendem também a encontrar mais oportunidades de interação, tendo, como decorrência, mais chances de aprimorar a sua compreensão dos estados mentais. A partir dessa perspectiva, entendese que a compreensão dos estados internos favorece e/ou é favorecida pela interaçáo com os pares, sendo esta uma questáo de interesse que necessita ser melhor investigada, visto que no estudo referido não foi obtida correlação entre a medida geral de compreensão emocional e aceitação social. Nessa direção, Souza (2008) destacou a relevância da realização de estudos contemplando as possíveis relaçóes entre a teoria da mente e outros aspectos do desenvolvimento infantil, como as habilidades sociais. $\mathrm{Na}$ visão da referida autora, a questão insere reflexóes a partir de duas direçóes: como a teoria da mente pode favorecer o desenvolvimento social; e de que forma as interaçóes sociais infantis contribuem para o desenvolvimento sociocognitivo. As investigaçôes realizadas até o momento, por se tratarem predominantemente de estudos correlacionais, não fornecem dados conclusivos, mas demonstram que teoria da mente e desenvolvimento social constituem aspectos intimamente relacionados.

Veiga e Miranda (2006), inspirando-se na visão modular da mente (e.g. Fodor, 1986), explicam que ela é constituída por um conjunto de módulos, sendo cada um específico e especializado em um tipo de processo ou atividade. Assim, seriam diferentes os módulos responsáveis pela percepção, orientação no espaço, linguagem, interação com as outras pessoas nas relaçóes sociais, entre outros. Desse modo, parece razoável admitir que, ao longo da evolução humana, foram surgindo estruturas cognitivas especializadas no manejo e na resoluçáo de problemas em âmbitos ou domínios muito distintos, destacando-se o mundo físico e o mundo social.

Como salientado por Astington (2004), o bebê vem ao mundo dotado de predisposiçóes ou programaçóes inatas que possibilitam diferenciar e classificar com rapidez, eficiência e pouco esforço as categorias do entorno, mais especificamente o mundo dos objetos e suas características e o mundo das pessoas e das interaçóes pessoais. Nesta perspectiva, os recém-nascidos já se mostram capazes de diferenciar os estímulos sociais dos não sociais. Contudo, como salientou a referida autora, para que os bebês possam avançar no conhecimento dos objetos e das pessoas, é necessário um longo aprendizado e muitas experiências que permitirão a eles compreender gradualmente que o ser humano apresenta um conjunto de particularidades, dentre as quais, a mente.

Para Baron-Cohen (2001) e Hughes e Leekam (2004), o desenvolvimento da teoria da mente representa uma grande conquista para a perpetuação da espécie, pois somente com o desenvolvimento dessa habilidade o homem tornou-se capaz de construir metas compartilhadas, dividir um mesmo foco de atenção, regular o seu comportamento, atender às demandas das interaçóes sociais, fazer trocas empáticas, ter açóes dirigidas, utilizar normas sociais para o controle emocional e, assim, desenvolver relaçôes sociais mais harmônicas e um repertório de resolução de problemas.

Partindo da premissa de que a teoria da mente coemerge com as relaçôes sociais estabelecidas pela criança no seu cotidiano, Lyra et al. (2008) consideram que tal habilidade é utilizada como uma "ferramenta" facilitadora para o estabelecimento e manutenção de suas relaçôes sociais. Nessa direção, diversos estudos indicam, por exemplo, a importância das relaçôes de amizade, relação entre irmãos e da aceitação social para o desenvolvimento sociocognitivo (Banerjee, Watling \& Caputi, 2011; Cutting \& Dunn, 2006; Dunn, Cutting \& Fisher, 2002; Slaughter, Dennis, \& Pritchard, 2002; Slomkowski \& Dunn, 1996).

Sabe-se ainda que o desenvolvimento de um indivíduo estáintimamenteligadoàs relaçôes próximas, especialmente às de amizades com os pares, que passam a constituir, ao longo desta trajetória desenvolvimental, um campo fértil de aprendizado para as crianças. Nessa perspectiva, assumem destaque investigaçóes longitudinais. Slomkowski e Dunn (1996), por 
exemplo, no intuito de analisar a compreensão social e a comunicação verbal naturalmente observadas entre amigos, realizaram um estudo longitudinal com 38 crianças pequenas que foram avaliadas em dois momentos. Tarefas de tomada de perspectiva afetiva e crença falsa referente ao conteúdo foram aplicadas quando elas tinham 3 anos e 4 meses de idade. A comunicação entre amigos (duração média dos episódios de conversação, brincadeira e jogos de faz de conta) foi codificada quando as crianças estavam com 3 anos e 11 meses de idade, a partir de dados observacionais. As performances em relação aos dois níveis etários de compreensão social apresentaramse significativamente associadas com a comunicação entre amigos, permitindo aos autores concluir que a comunicação social está relacionada à capacidade de compreender e prever a ação do outro.

Maguire e Dunn (1997) buscaram, por meio de um estudo transversal, fornecer dados descritivos sobre as diferenças individuais de crianças pequenas nas relaçóes próximas de amizade e examinar tais diferenças e a compreensão das crianças sobre as emoçóes e os estados mentais dos outros. Foram observadas as interaçôes infantis durante a brincadeira e realizadas avaliaçóes sociocognitivas (tarefas de compreensão das emoções e crença falsa de conteúdo) em 41 crianças de 6 a 7 anos, juntamente com amigos próximos da mesma faixa etária. Os dados observacionais não permitem indicar uma associação entre as relaçóes amistosas durante o brincar e a coordenação da brincadeira, pois ocorreram interaçôes positivas entre as crianças independente de uma delas assumir a coordenaçáo da atividade. Os resultados sugerem relação entre o desenvolvimento da compreensão social e as experiências vivenciadas durante as brincadeiras infantis.

Com o objetivo de investigar o desenvolvimento da compreensão infantil da mente, da emoção e da utilização de termos mentais nas conversaçôes com os amigos, Hughes e Dunn (1998) acompanharam longitudinalmente 50 crianças $(25$ pares de amigos; 25 meninas e 25 meninos) de 3 a 5 anos. Os participantes foram avaliados por meio de diversas tarefas de crença falsa (local inesperado, conteúdo enganoso), compreensão emocional (tarefa de identificação da emoção de personagens) e tomada de perspectiva (identificação e exploração das possíveis causas da emoçáo apresentada pelo personagem). Foram realizadas filmagens enquanto elas brincavam em uma sala previamente preparada com brinquedos e fantasias. Evidenciou-se melhora expressiva no que tange ao desempenho da teoria da mente (crença falsa e compreensão das emoçóes) e de tomada de perspectiva afetiva no decorrer dos 13 meses de estudo. Além disso, ambas as variáveis correlacionaram-se ao uso de termos mentais durante as interações infantis. Os participantes que utilizaram mais termos mentais durante as conversaçóes com os amigos apresentaram resultados mais positivos no que se refere à avaliação da crença falsa, sendo esta também relacionada à compreensão emocional. Tais resultados indicam que as crianças que estabeleciam conversaçóes mais ricas entre os pares apresentavam maior sofisticação quanto ao desenvolvimento sociocognitivo, sustentando, portanto, a relevância das relaçôes de amizade.

Tendo em vista que essas relaçóes são fundamentais para o desenvolvimento sociocognitivo, Dunn et al. (2002) realizaram uma pesquisa longitudinal envolvendo 70 crianças (inicialmente com 4 anos) que foram acompanhadas até a transição para a escola básica. O propósito dos autores era verificar se as relaçóes de amizade estavam relacionadas à compreensão social, às características dos seus amigos, à qualidade da relação de amizade, aos preditores de insight, experiências compartilhadas de faz de conta e ao nível educacional das mães.

O vocabulário infantil foi mensurado pela British Picture Vocabulary Scale (BPVS). Já a compreensão social foi avaliada por meio de testes de cognição social (tarefas de crença falsa de local inesperado, tomada de perspectiva e compreensão das emoções mistas), observação diádica de jogos entre pares, avaliação docente do comportamento pró-social infantil (Strengths and Difficulties Questionnaire - SDQ) e entrevistas com as crianças sobre as suas amizades. Verificou-se que os constructos estavam relacionados com a perspectiva de fazer, gostar e manter os amigos da escola.

Para analisar a hipótese de que a teoria da mente de crianças pré-escolares relaciona-se com seus níveis de aceitação pelos pares, Slaughter et al. (2002) realizaram dois estudos transversais. No primeiro, 78 crianças com 4 a 6 anos de idade responderam a diferentes tarefas de teoria da mente (histórias envolvendo situaçóes de crença falsa, desejos diferentes e emoçóes conflitantes) e participaram de uma entrevista individual que objetivou avaliar a aceitação social, sendo focalizada a escolha de amigos para brincar. Os resultados mostraram relação significativa entre teoria da mente e preferência social 
em uma subamostra de crianças com 5 anos de idade, constatando-se que as mais populares apresentaram escore mais elevado nas tarefas de teoria da mente do que as identificadas como rejeitadas.

O segundo estudo de Slaughter et al. (2002) foi uma replicação e ampliação do primeiro, mediante uma amostra de 87 crianças e a inclusão de medidas de avaliação da teoria da mente, mais especificamente da compreensão da crença falsa (local inesperado) e inteligência verbal medida por meio do Peabody Picture Vocabulary Test - Revised (PPVT), além de avaliações dos professores sobre comportamentos pró-social e agressivos por um questionário. Os resultados mostraram que o comportamento pró-social foi o melhor preditor do escore de preferência social, ou seja, as crianças que eram bem aceitas pelos seus pares foram classificadas como pró-sociais e não agressivas pelos seus professores. Quando a amostra do segundo estudo foi dividida em grupos etários, a habilidade de teoria da mente foi verificada como o melhor preditor de preferência social para as mais velhas (por volta de 5 anos), enquanto os comportamentos agressivos e pró-sociais foram os melhores preditores para a aceitação entre os pares para as crianças mais novas. No geral, o padrão dos resultados sugere que o impacto da habilidade de teoria da mente na aceitação entre os pares é modesto, mas aumenta com a idade.

Para avaliar se a interação entre os pares está ligada ao desenvolvimento da compreensão da mente e como essa associação reflete nos diferentes relacionamentos, Cutting e Dunn (2006) realizaram uma investigação com 43 crianças de 4 anos de idade, que foram observadas interagindo com um irmão e um amigo próximo com a utilização de testes sociocognitivos (tarefas contendo histórias e perguntas sobre a compreensão da crença falsa e das emoçóes) e de linguagem (BPVS). Os professores e os pais foram entrevistados sobre a qualidade da relação das crianças com seus pares e irmãos, respectivamente. A família forneceu informaçóes adicionais quanto à realidade socioeconômica. E os resultados indicaram relação entre as habilidades sociocognitivas, a comunicação bem sucedida entre irmáos e amigos e a inserção em jogos de faz de conta nos dois relacionamentos (irmão e amigo próximo). A habilidade linguística e as condiçóes socioeconômicas foram importantes somente na interação (conversação e brincadeiras) com amigos, mas não com irmãos.
A relação entre os pares e o entendimento de gafes foram examinados em um estudo longitudinal realizado por Banerjee et al. (2011) mediante o acompanhamento de 210 crianças de 5 a 6 anos e 8 a 9 anos. Utilizaram-se tarefas e testes para avaliar o entendimento envolvendo a gafe, mediante histórias e perguntas dirigidas a investigar a capacidade infantil de identificar que algo foi dito em uma situação inadequada; a aceitação e a rejeição entre os pares (cada criança foi indagada sobre quais colegas tem maior ou menor preferência), além do vocabulário receptivo medido pela BPVS. Constatou-se, a partir do acompanhamento longitudinal dos participantes, que a rejeição pelos pares pode prejudicar a aquisição de entendimento de gafes, visto que os participantes com menor aceitação social apresentavam desempenhos inferiores quanto à compreensão delas. Esses dados permitem considerar a existência de associaçôes importantes e complexas entre a compreensão social e o relacionamento com os colegas na infância.

Essas evidências empíricas indicam que a teoria da mente é notadamente relevante para a inserção da criança no mundo social, na medida em que se relaciona com a manutenção e o êxito das relaçôes sociais (Hughes \& Leekam, 2004; Lyra et al., 2008; Pires, 2010). Além disso, as conclusões obtidas sugerem que crianças que têm compreensão mais acurada das crenças, desejos e emoçôes apresentam maior aceitaçâo social (Slaughter et al., 2002). A relevância da adaptação psicossocial para a trajetória infantil tem motivado pesquisadores a investigarem a relação entre aspectos do desenvolvimento social, tais como amizade e a compreensão dos estados mentais.

A partir dos estudos mencionados, verifica-se que a relação entre teoria da mente e desenvolvimento social pode constituir uma via de máo dupla. Tal afirmação encontra apoio em pelo menos dois eixos de análise. Primeiramente, torna-se necessário considerar que as investigaçóes já realizadas sugerem que o desenvolvimento social pode funcionar como um estímulo à teoria da mente, na medida em que possibilita às crianças o acesso aos estados mentais ou à relação entre eles e a conduta social, dandose extrema relevância ao contexto em que a criança está inserida (tamanho da família, estilo parental, ambiente escolar, amigos, dentre outros). De outro ponto de vista, os dados empíricos indicam também que uma teoria da mente bem desenvolvida pode favorecer o desenvolvimento social, visto que o seu entendimento exerce influência na maneira como as 
crianças interagem, na qualidade das suas relaçóes de amizade e em sua compreensão social. Pesquisadores (e.g. Banerjee et al., 2011; Cutting \& Dunn, 2006; Rodrigues \& Pires, 2010; Souza, 2008) têm salientado a necessidade de novos estudos para esclarecer qual seria a direção e, até mesmo, uma possível bidireção da relação entre teoria da mente e desenvolvimento social.

\section{Teoria da Mente, Aceitação Social e Pró-sociabilidade na Infância}

Alguns estudos têm contribuído para ampliar a compreensão da interface entre o entendimento de crenças, desejos e emoçóes próprias e alheias e importantes variáveis do desenvolvimento social, tais como a aceitação social e a pró-sociabilidade. Encontram-se, na literatura internacional, estudos envolvendo a relação entre o desenvolvimento da teoria da mente e aspectos como a avaliação pelos pares, a manifestação de açóes pró-sociais e a agressividade infantil (e.g. Cassidy et al., 2003; Denham et al., 2002). Apesar desse escopo de pesquisas ser ainda recente, já são encontrados trabalhos nessa direção.

Watson et al. (1999), por exemplo, realizaram dois estudos transversais com o objetivo de explorar a relação entre habilidades sociais e teoria da mente em crianças pequenas. No primeiro, 26 crianças (3 a 6 anos) foram avaliadas por meio do teste de linguagem Test for the Auditory Comprehension of Language - Revised (TACL-R), tarefas de crença falsa de conteúdo e um questionário para avaliação das habilidades sociais infantis nas interaçóes com seus pares, preenchido pelo professor. Os resultados apontaram correlação entre o desempenho nas tarefas de crença falsa e a avaliação das habilidades sociais realizada pelo professor. Os resultados do segundo trabalho, que envolveu 52 crianças (3 a 6 anos) e manteve os mesmos instrumentos, com exceção da avaliaçáo docente, realizada mediante adaptação de um instrumento padronizado (Perceived Competence Scale for Children), indicaram convergência com os dados do primeiro estudo, reforçando a premissa de que a teoria da mente mostra-se especialmente importante para as interaçóes sociais.

Badenes, Estevan e Bacete (2000) objetivaram investigar, em um estudo transversal, se as experiências sociais fomentadas entre os pares associavam-se ao desenvolvimento da teoria da mente em 313 crianças, entre 4 a 6 anos de idade. Elas foram analisadas por meio de um instrumento de avaliação sociométrica (cada criança relatava por quais colegas apresentava maior ou menor preferência), avaliação do comportamento social por meio do Pupil Evaluation Inventory (PEI), tarefas de teoria da mente (histórias e perguntas dirigidas a investigar a compreensão da crença falsa infantil), testes voltados à linguagem figurativa (12 Strange Stories from Happé) e uma tarefa para medir a compreensão das emoçôes (identificação da emoção vivenciada por personagens de uma história). Os resultados não evidenciaram, contudo, diferenças quanto ao desempenho de crianças rejeitadas pelo grupo de pares quando comparadas às demais no que se refere à teoria da mente. Esses dados podem indicar que crianças com pouca aceitação social não apresentam déficits sociocognitivos, porém, é possível pensar que estas possuam uma forma diferenciada de lançar mão da compreensão dos estados mentais alheios. Mais especificamente, apesar de compreenderem os estados mentais alheios, em suas interaçóes sociais tais crianças nem sempre expressam tal entendimento na manifestação de comportamentos socialmente adequados.

O estudo longitudinal de Denham et al. (2002) focalizou a relação entre a compreensão das emoçóes e agressividade com os pares em crianças pré-escolares. Participaram 127 indivíduos, com 3 e 4 anos de idade à época da primeira avaliaçáo. A coleta dos dados ocorreu em três momentos: com o uso de três medidas diferenciadas para mensuração da compreensão das emoçôes; observação naturalística das crianças em interaçóes com os pares; e aplicação de um questionário que avaliou a competência social a partir da ótica do professor.

O método observacional e o questionário respondido pelos docentes buscaram delimitar, dentre os participantes, o grupo com maior incidência de comportamento agressivo. Os achados indicam que o conhecimento das emoçóes avaliadas inicialmente (3 e 4 anos) prediz manifestaçóes de agressividade posterior, ou seja, crianças com baixo desempenho na avaliação da compreensão emocional apresentaram maior incidência de comportamentos agressivos no futuro. Para os pesquisadores, esse dado é condizente com a literatura, visto que a criança precisa compreender as emoçóes para desenvolver outras habilidades importantes para o êxito nas relaçóes sociais, tais como a autorregulaçáo emocional e o gerenciamento de conflitos.

Cassidy et al. (2003) estudaram uma amostra de 67 indivíduos (3 a 6 anos) no intuito de pesquisar 
a relação entre teoria da mente e comportamentos pró-sociais em pré-escolares. As avaliaçôes incidiram sobre tarefas de teoria da mente (crença falsa e decepção), compreensão das emoçôes (tomada de perspectiva e emoçáo real-aparente) e competência social relatada pelos pares investigada por meio de uma avaliaçáo sociométrica. Utilizou-se também o Test of Early Language Development - 2 (TELD-2) para aferição do vocabulário infantil, sendo também realizada a observação dos comportamentos infantis em sala de aula. A ótica do professor foi investigada mediante a aplicação da escala Social Skills Rating System - Preschool Form. O desempenho em teoria da mente foi positivamente correlacionado à avaliação da competência social pelo professor e pelos pares e aos comportamentos observados em classe, e os comportamentos pró-sociais mais frequentemente identificados foram os de cooperação, ajuda e partilha. As correlaçóes sugerem que as avaliaçóes realizadas pelos pares, professores e por meio da observaçáo foram condizentes com a individual da teoria da mente. Tais achados reforçam a relevância da capacidade de compreender crenças, desejos, intenções e emoçôes próprias e alheias para o êxito nas relaçôes sociais.

Machado et al. (2008) realizaram um estudo com 40 crianças (5 e 6 anos) para avaliar se a compreensão das emoçóes constitui fator de mediação entre as habilidades acadêmicas e a aceitação pelos pares. Analisou-se também se o entendimento emocional compõe um fator de mediação para a associação entre competência social e aceitação pelos pares com a aplicaçáo de um teste de conhecimento das emoçóes com as crianças e uma escala de avaliação da competência social, respondidos pelos pais. Os resultados mostraram que essa compreensão emocional constitui fator de mediação nas associaçôes entre as competências acadêmicas e a aceitação pelos pares. Contudo, não foram obtidos dados significativos com relação à ligação entre conhecimento emocional, competência social e a aceitação em questão.

Pavarini e Souza (2010) investigaram se a aquisição da teoria da mente associa-se à habilidade de compartilhar emoções e à motivação pró-social nos anos pré-escolares. Trinta e sete crianças ( 4 a 6 anos de idade) responderam as tarefas de crença e emoçáo e emoção real-aparente, extraídas da escala de teoria da mente de Wellman e Liu (2004) e um instrumento de avaliação da empatia elaborado para o estudo, baseado em vídeos apresentados para cada um dos participantes, que era questionado quanto às emoçóes vivenciadas pelos personagens. Os resultados não evidenciaram associaçáo significativa entre o desempenho nas tarefas de teoria da mente e o grau de empatia das crianças. Contudo, uma correlação entre a motivação pró-social e as tarefas foi obtida. As autoras argumentam que a compreensão dos estados mentais pode não constituir um requisito para a manifestação empática, mas tende a favorecer a manifestação do comportamento pró-social em crianças pré-escolares, traduzindo uma questão que necessita ser melhor investigada no contexto nacional.

Rodrigues e Ribeiro (2011) realizaram um estudo de seguimento para avaliar diferenças no grau de empatia em dois grupos de crianças, participantes e náo participantes de um programa de intervenção conduzido pelo professor em sala de aula, que objetivou promover o desenvolvimento sociocognitivo por meio da leitura mediada de livros de histórias infantis. Participaram 40 crianças, com idade média de 7 anos (20 participantes e 20 crianças que não participaram do referido programa). Os resultados evidenciaram que o grupo participante apresentou médias significativamente mais expressivas quanto ao grau de empatia quando comparadas aos indivíduos náo participantes, sugerindo que o trabalho implementado favoreceu indiretamente $o$ desenvolvimento da empatia nas crianças.

Caputi, Lecce, Pagnin e Banerjee (2012) acompanharam 70 indivíduos, de 5 a 7 anos, que passaram por avaliações anuais em relaçáo à teoria da mente (tarefas de crenças falsas e emoçóes mistas), compreensão das emoções, e pró-sociabilidade, mensurada a partir da aplicação da subescala cooperatividade do Social Skills Rating System, de cinco itens da Pro-social Scale of the SDQ e sete itens da Prosocial With Peers Subscale of the CBS. A nomeação pelos pares foi realizada a partir de uma avaliação sociométrica, e a aferição da habilidade verbal por meio do Peabody Picture Vocabulary Test - Revised e Test for Reception of Grammar (TROG). Objetivouse verificar como tais habilidades se associavam na transição da criança da educação infantil para o ensino fundamental. $\mathrm{O}$ desempenho em teoria da mente aos 5 anos associou-se às manifestaçôes pró-sociais aos 6 e 7 anos. Além disso, o comportamento pró-social foi preditor das relaçóes com os pares, sugerindo que interaçóes sociais positivas podem reduzir o risco de rejeição na infância. No entanto, teoria da mente e comportamento pró-social não se associaram de 
maneira consistente, sugerindo a necessidade de estudos futuros que possam incluir fatores não investigados, como o contexto familiar, por exemplo.

Pavarini et al. (2011) pesquisaram no âmbito nacional as possíveis correlações entre compreensão das emoções, aceitação social e avaliação de atributos comportamentais pelos pares em um grupo de 52 crianças (8 a 10 anos). Foram aplicados dois instrumentos: Teste de Inteligência Emocional (TIEC) e avaliação sociométrica pelos pares. Os resultados não mostraram associação entre compreensão das emoçôes e aceitação social. Obteve-se correlação negativa entre a avaliação de atributos ligados à agressividade e compreensão emocional. As autoras concluíram que o conhecimento das diferentes emoçôes, bem como a autorregulação emocional, pode favorecer a diminuiçâo do comportamento agressivo.

Ribas (2011) comparou o desempenho em teoria da mente e compreensão das emoções entre crianças que apresentavam indícios de comportamento agressivo e as que não apresentavam tais indicadores. $\mathrm{Na}$ primeira etapa do estudo, os professores responderam um instrumento dirigido a avaliar a percepção dos comportamentos agressivos de 115 crianças (6 e 7 anos). Os dados permitiram delimitar 60 delas - 30 identificadas como socialmente competentes e 30 com indícios de comportamentos agressivos. Elas participaram da segunda etapa do estudo respondendo a escala de teoria da mente adaptada para o contexto nacional por Domingues, Valério, Panciera e Maluf (2007) e o TIEC. Os resultados evidenciaram relação entre teoria da mente e desenvolvimento social. As crianças descritas como socialmente competentes apresentaram melhor desempenho nas tarefas de teoria da mente e no teste de inteligência emocional, se comparadas ao grupo com indícios de comportamento agressivo, sugerindo importantes implicações teórico-práticas.

A partir dos estudos apresentados e da diversidade dos objetivos e metodologias, pode-se considerar, com os resultados obtidos, a possibilidade de favorecer o desenvolvimento da teoria da mente, por meio de pesquisas com intervenção e, a partir disso, a pró-sociabilidade infantil, por exemplo.

Os estudos aqui descritos fornecem uma compreensão mais ampla da relevância da compreensão dos estados mentais para a trajetória de desenvolvimento infantil. Os resultados reportados, oriundos, em sua expressiva maioria, de estudos internacionais, indicam que o desenvolvimento da teoria da mente tem relação positiva com o comportamento pró-social e com a competência social. No tocante à compreensão das emoções, um dos componentes da teoria da mente, verificar-se que o conhecimento das emoçóes pode favorecer tanto o melhor desempenho nas interaçóes sociais quanto contribuir para a diminuição do comportamento agressivo infantil.

\section{Consideraçóes Finais}

Os estudos aqui apresentados indicam que o desenvolvimento social pode fomentar o da teoria da mente, ao mesmo tempo em que uma teoria da mente bem desenvolvida pode favorecer o desenvolvimento social. A direção dessa associação e, até mesmo, uma possível bidirecionalidade, como já foi dito, ainda não estão claras na literatura, visto que os estudos contemplando tal relação são predominantemente de natureza correlacional. Trabalhos futuros poderáo fornecer dados mais conclusivos.

No que se refere aos delineamentos utilizados, encontrou-se um expressivo contingente de estudos transversais, mas é possível verificar também a realização de investigaçôes longitudinais, realidade promissora para a área da psicologia do desenvolvimento, dado que a compreensáo dos estados mentais ocorre de forma gradual na infância. Em relação aos instrumentos utilizados, constatase que o desenvolvimento da teoria da mente é, na grande parte dos estudos, aferida por meio de tarefas que contemplam aspectos como crenças e emoçôes. Destaca-se que a escala de Wellman e Liu (2004), como construiu-se mais recentemente, foi utilizada apenas em dois estudos, e vale ressaltar que é importante ampliar as pesquisas referentes a esse instrumento. Testes de compreensão das emoçóes e de aferição da linguagem também foram utilizados de maneira recorrente. Quanto aos diversos aspectos do desenvolvimento social, questionários preenchidos pelos professores têm sido bastante considerados, sendo ainda comum a presença de observaçóes naturalísticas e avaliação pelos pares. Salienta-se que, no contexto nacional, encontra-se uma escassez de instrumentos voltados para avaliação de aspectos referentes à competência social infantil, constituindo ainda um desafio aos pesquisadores brasileiros.

Os dados empíricos aqui reportados permitem indicar a importância das relaçôes interpessoais na infância e da aceitação social para o desenvolvimento 
infantil. Nesse sentido, torna-se ainda mais pertinente ampliar o escopo das investigaçóes na realidade nacional, visto que grande parte do conhecimento científico dessa área provém da literatura internacional.

\section{Referências}

Astington, J. W. (2004). The child's discovery of the mind. Cambridge: Harvard University Press.

Astington, J. W., \& Jenkins, J. M. (1999). A longitudinal study of the relation between language and theory-of-mind development. Developmental Pshychology, 35(5), 1311-1320.

Badenes, L. V., Estevan, R. A. C., \& Bacete, F. J. G. (2000). Theory of mind and peer rejection at school. Social Development, 9(3), 271-283.

Banerjee, R., Watling, D., \& Caputi, M. (2011). Peer relations and the understanding of faux pas: longitudinal evidence for bidirectional associations. Child Development, 82, 1887-1905.

Baron-Cohen, S. (2001). Theory of mind in normal development and autism. Prisme, 34, 174-183.

Baron-Cohen, S., Leslie, A. M., \& Frith, U. (1985). Does the autistic child have a "theory of mind"? Cognition, 21(1), 37-46.

Caputi, M., Lecce, S., Pagnin, A., \& Banerjee, R. (2012). Longitudinal effects of theory of mind on later peer relations: the role of prosocial behavior. Developmental Psychology, 48(1), 257-270.

Cassidy, K. H., Werner, R. S., Rourke M., Zubernis, L. S., \& Balaraman, G. (2003). The relationship between psychological understanding and positive social behaviors. Social Development, 12(2), 198-221.

Cutting, A. L., \& Dunn, J. (2006). Conversations with siblings and with friends: links between relationship quality and social understanding. British Journal of Developmental Psychology, 24(1), 73-87.

Denham, S. A., Caverly, S., Schmidt, M., Blair, K., DeMulder, E., Caal, et al. (2002). Preschool understanding of emotions: contributions to classroom anger and aggression. Journal of Child Psychology and Psychiatry, 43(7), 901-916.

Dias, M. G. B. B. (1993). O desenvolvimento do conhecimento da criança sobre a mente. Psicologia: Teoria e Pesquisa, 9, 587-600.

Dias, M. G. B. B., Soares, G. B., \& Sá, T. P. (1994). Conhecimento sobre a mente e compreensão sobre as intençôes do experimentador. Psicologia: Teoria e Pesquisa, 10, 221-229.
Domingues, S. F. S., Valério, A., Panciera, S. D. P., \& Maluf, M. R. (2007). Tarefas de crença falsa na avaliação de atribuição de estados mentais de crença. In P. W. Schelini (Org.), Alguns domínios da avaliação psicológica, (pp. 141-162). Campinas: Alínea.

Dunn, J., Cutting, A. L., \& Fisher, N. (2002). Old friends, new friends: predictors of children's perspectives on their friends at school. Child Development, 73(2), 621-635.

Flavell, J. H., Flavell, E. R., \& Green, F. L. (1983). Development of the appearance - reality distinction. Cognitive Psychology, 15(1), 95-120.

Flavell, J. H., Miller, P. H., \& Miller, S. A. (1999). Desenvolvimento cognitivo. Porto Alegre: Artes Médicas.

Fodor, J. (1986). La modularidad de la mente. Madrid: Editora Morata.

Harris, P. L., Rosnay, M., \& Pons, F. (2005). Language and children's understanding of mental states. Current Directions in Psychological Science, 14(1), 69-73.

Hughes, C., \& Dunn, J. (1998). Understanding mind and emotion understanding: longitudinal associations with mental-state talk between young friends. Developmental Psychology, 34(5), 1026-1037.

Hughes, C., \& Leekam, S. (2004). What are the links between theory of mind and social relations? Review, reflections and new directions for studies of typical and atypical development. Social Development, 13(4), 590-619.

Lyra, P., Roazzi, A., \& Garvey, A. (2008). Emergência da teoria da mente em relaçôes sociais. In T. M. Sperb \& M. R. Maluf (Orgs.), Desenvolvimento sociocognitivo: estudos brasileiros sobre "teoria da mente”, (pp. 55-92). São Paulo: Vetor.

Machado, P., Veríssimo, M., Torres, N., Peceguina, I., Santos, A. J., \& Rolão, T. (2008). Relaçôes entre o conhecimento das emoçóes, as competências académicas, as competências sociais e a aceitação entre pares. Análise Psicológica, 3, 463-478.

Maguire, M. C., \& Dunn, J. (1997). Friendships in early childhood, and social understanding. International Journal of Behavioral Development, 21(4), 669-686.

Pavarini, G., \& Souza, D. H. (2010). Teoria da mente, empatia e motivação pró-social em crianças préescolares. Psicologia em Estudo, 15(3), 613-622. 
Pavarini, G., Loureiro, C. P., \& Souza, D. H. (2011). Compreensão de emoçóes, aceitação social e avaliação de atributos comportamentais em crianças escolares. Psicologia: Reflexão e Crítica, 24(1), 135-143.

Pires, L. G. (2010). Evocação de termos mentais na leitura de diferentes livros de imagens: um estudo com pré-escolares. Dissertação de Mestrado não publicada. Programa de Pós-Graduação em Psicologia da Universidade Federal de Juiz de Fora, Juiz de Fora.

Ribas, D. A. (2011). Teoria da mente e compreensão das emoçôes: um estudo comparativo com alunos do ensino fundamental. Dissertação de Mestrado não publicada. Programa de Pós-Graduação em Psicologia da Universidade Federal de Juiz de Fora, Juiz de Fora.

Rodrigues, M. C., \& Pires, L. G. (2010). Teoria da mente: linguagem e contextos de desenvolvimento infantil. In M. C. Rodrigues \& T. M. Sperb (Orgs), Contextos de desenvolvimento da linguagem, (pp. 103-135). São Paulo: Vetor.

Rodrigues, M. C., \& Ribeiro, N. N. (2011). Avaliação da empatia em crianças participantes e não participantes de um programa de desenvolvimento sociocognitivo. Psicologia - Teoria e Prática, 13(2), 114-126.

Slaughter, V., Dennis, M. J., \& Pritchard, M. (2002). Theory of mind and peer acceptance in preschool children. British Journal of Developmental Psychology, 20(4), 545-564.

Slomkowski, C., \& Dunn, J. (1996). Young children's understanding of other people's beliefs and feelings and their connected communication with friends. Developmental Psychology, 32(3), 442-447.

Souza, D. H. (2008). De onde e para onde? As interfaces entre linguagem, teoria da mente e desenvolvimento social. In T. M. Sperb \& M. R. Maluf (Orgs.), Desenvolvimento sociocognitivo: estudos brasileiros sobre "teoria da mente", (pp. 3354). Sáo Paulo: Vetor.

Veiga, E. C., \& Miranda, V. R. (2006). A importância das inteligências intrapessoal e interpessoal no papel dos profissionais da área de saúde. Ciências e Cognição, 9, 64-72.

Watson, A. C., Nixon, C. L, Wilson, A., \& Capage, L. (1999). Social interaction skills and theory of mind in young children. Developmental Psychology, 35(2), 386-391.
Wellman, H. M., \& Liu, D. (2004). Scaling of theory-of-mind tasks. Child Development, 75(2), 523-541.

Wimmer, H., \& Perner, J. (1983). Beliefs about beliefs: representation and constraining function of wrong beliefs in young children's understanding of deception. Cognition, 13(1), 103-128.

\section{Endereço para correspondência:}

Renata de Lourdes Miguel da Silva

Rua João de Araújo Braga, 643

CEP 36070-650 - Juiz de Fora/ MG

E-mail: renata.miguel@yahoo.com.br

Recebido em 14/05/2012

Revisto em 30/09/2012

Aceito em 29/10/2012 